\title{
SELF-HOMOTOPY-EQUIVALENCES OF A SPACE WITH TWO NONVANISHING HOMOTOPY GROUPS
}

\author{
KOUZOU TSUKIYAMA ${ }^{1}$
}

\begin{abstract}
The group $G_{\#}(X)$ is isomorphic to $H^{n}\left(\pi_{1}(X) ; \pi_{n}(X)\right)$ for the connected CW-complex $X$ with two nonvanishing homotopy groups $\pi_{1}(X), \pi_{n}(X)$, where $\pi_{1}(X)$ acts on $\pi_{n}(X)$ usually.
\end{abstract}

0. Introduction. Let $X$ be a connected CW-complex with base point *. Then we have considered the group $G_{\#}(X)$ of all based homotopy classes of self-homotopyequivalences of $X$ inducing the identity automorphisms of all homotopy groups (cf. [1], [11], [12]).

In this note, we show that $G_{\#}(X)=H^{j}\left(K\left(\pi_{i}(X), i\right) ; \pi_{j}(X)\right)$ for the connected CW-complex $X$ with two nonvanishing homotopy groups $\pi_{i}(X), \pi_{j}(X)(j>i)$, where $\pi_{i}(X)$ acts on $\pi_{j}(X)$ usually if $i=1$ [Theorem 2.2].

This implies that to determine the group $G_{\#}(X)$ is to compute the (local coefficient) cohomology of the group $\pi_{i}(X)$, that is, a problem in group theory.

For the simply connected case, the above result is shown by W. Shih [10] and later by Y. Nomura [8].

The method of the proof is different from that of the simply connected case.

Some examples in $G_{\#}(X)=1$ are discussed in [11] and other examples can be found in [3], [4] and [2].

In $\$ 1$ we review J. F. McClendon [7] and the author [12], and in $\$ 2$ we prove the above theorem; in $\$ 3$ some examples of $G_{\#}(X)$ are given.

Throughout this note, all spaces are base-pointed, and homotopies preserve base points, and we use the terminology of [12].

1. Twisted principal fibrations. Let $\phi: \pi_{1}(B) \rightarrow$ Aut $G$ be the local coefficient system on $B$, and $p: \bar{P}_{\theta} \rightarrow B$ be the twisted principal fibration with fibre $K(G, n)$ (cf. [12, pp. 1-2]).

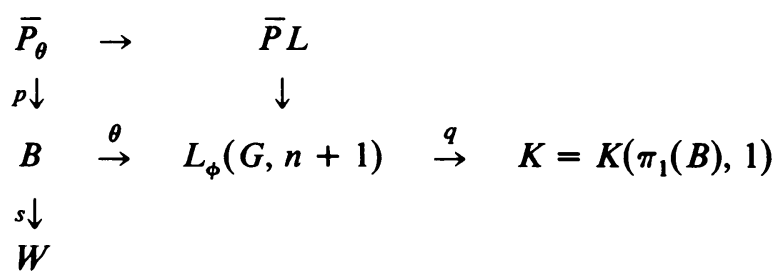

Received by the editors March 6, 1978 and, in revised form, February 21, 1979.

AMS (MOS) subject classifications (1970). Primary 55B25, 55D10, 55F05, 55G45.

Key words and phrases. Homotopy equivalence, twisted principal fibration, local coefficient cohomology.

'This research was supported by NSF Grant 274018 in Japan.

(C) 1980 American Mathematical Society $0002-9939 / 80 / 0000-0226 / \$ 02.25$ 
Associated with this diagram, we have the following.

Proposition 1.2 ([7, p. 42]). The following sequence is exact:

$$
\left[\bar{P}_{\theta}, \hat{\Omega}_{W} B\right]_{B} \stackrel{\delta}{\rightarrow} H^{n}\left(\bar{P}_{\theta} ; G\right) \stackrel{\gamma}{\rightarrow}\left[\bar{P}_{\theta}, \bar{P}_{\theta}\right]_{W} \stackrel{p^{*}}{\rightarrow}\left[\bar{P}_{\theta}, B\right]_{W}
$$

In this sequence, $\hat{\Omega}_{W} B=F\left(S^{1}, B \mid s\right)=\left\{\omega: S^{1} \rightarrow B \mid s \omega\right.$ is constant $\},[,]_{W}$ and $[,]_{B}$ denote the $W$-homotopy and $B$-homotopy sets, respectively, and $H^{n}\left(\bar{P}_{\theta} ; G\right)$ is the cohomology with the local coefficient induced by

$$
q \theta p: \bar{P}_{\theta} \rightarrow B \rightarrow L_{\phi}(G, n+1) \rightarrow K=K\left(\pi_{1}(B), 1\right) .
$$

There is an action $\nu_{*}: H^{n}\left(\bar{P}_{\theta} ; G\right) \times\left[\bar{P}_{\theta}, \bar{P}_{\theta}\right]_{W} \rightarrow\left[\bar{P}_{\theta}, \bar{P}_{\theta}\right]_{W}$ (cf. [12, p. 3, (1.8)]), and we denote the isotropy group of the identity map $1_{\bar{P}_{\theta}}: \bar{P}_{\theta} \rightarrow \bar{P}_{\theta}$ by $I\left(1_{\bar{P}_{\theta}}\right)$.

The map $\delta$ of Proposition 1.2 implies the following.

Proposition 1.3 ([7, Theorem 3.4]). $\operatorname{Im} \delta=I\left(1_{\overline{P_{f}}}\right)$.

Put $W=K$ and $s=q \theta$ in the diagram (1.1). Then we have the following associated with the group $G_{\#}(X)$.

Proposition 1.4 ([12, Theorem 3.6]). Assume that a connected $C W$-complex $B$ satisfies $\pi_{i}(B)=0$ for every $i \geqslant n$. Let $\phi: \pi_{1}(B) \rightarrow$ Aut $G$ be the local coefficient system on $B$, and $p: \bar{P}_{\theta} \rightarrow B$ be the twisted principal fibration with fibre $K(G, n)$. Then, the following sequence of groups and homomorphisms is exact.

$$
1 \rightarrow I\left(1_{\bar{P}_{\theta}}\right) \stackrel{\subset}{\rightarrow} H^{n}(B ; G) \stackrel{\Delta}{\rightarrow} G_{\#}\left(\bar{P}_{\theta}\right) \stackrel{J}{\rightarrow} G_{\#}(B),
$$

where $H^{n}(B ; G)=H^{n}\left(\bar{P}_{\theta} ; G\right)$ is the cohomology with the local coefficient induced by

$$
q \theta: B \rightarrow L_{\phi}(G, n+1) \rightarrow K=K\left(\pi_{1}(B), 1\right),
$$

$\Delta(a)=\nu_{*}\left(a, 1_{\bar{P}_{0}}\right)$ and $J$ is a natural homomorphism (cf. $\left.[12, p p .4-5]\right)$.

Considering a connected CW-complex $X$ and its Postnikov-system $\left\{X_{n}\right\}$, let $\phi$ : $\pi_{1}\left(X_{n-1}\right)=\pi_{1}(X) \rightarrow$ Aut $\pi_{n}(X)$ be the usual local coefficient system on $X_{n-1}$, and put $B=X_{n-1}, W=K\left(\pi_{1}(X), 1\right)=K$ and $s=p_{2} \circ \ldots{ }^{\circ} p_{n-2} p_{n-1}: X_{n-1} \rightarrow X_{1}$ $=K\left(\pi_{1}(X), 1\right)$ in diagram (1.1). Associated with the fibration $p_{n}: X_{n} \rightarrow X_{n-1}$, let

$$
[k] \in\left[X_{n-1}, L_{\phi}\left(\pi_{n}(X), n+1\right)\right]_{K}=H^{n+1}\left(X_{n-1} ; \pi_{n}(X)\right)
$$

be the transgression image of the identity map $i_{n} \in[F, F]^{\phi}$, where $F=K\left(\pi_{n}(X), n\right)$ and $[F, F]^{\tilde{\phi}}$ denotes the equivariant homotopy classes under the action $\phi$ of $\pi_{1}(X)$ on $\pi_{n}(X)$ (cf. [6, p. 3]). We have a fibration $\bar{P}_{k} \rightarrow X_{n-1}$, then there exists a based homotopy equivalence $\eta: X_{n} \rightarrow \bar{P}_{k}$, which is a fibre map over $X_{n-1}[12$, Proposition 2.1]. And we have the following.

Proposition 1.5 ([12, Corollary 3.7]). Let $X$ be a connected $C W$-complex and $\left\{X_{n}\right\}$ be a Postnikov-system of $X$. Then, the following sequence of groups and homomorphisms is exact for every $n>1$.

$$
1 \rightarrow I\left(1_{X_{n}}\right) \stackrel{\subset}{\rightarrow} H^{n}\left(X_{n-1} ; \pi_{n}(X)\right) \stackrel{\Delta}{\rightarrow} G_{\#}\left(X_{n}\right) \stackrel{J}{\rightarrow} G_{\#}\left(X_{n-1}\right)
$$


where $I\left(1_{X_{n}}\right)=I\left(1_{\bar{P}_{k}}\right), H^{n}\left(X_{n-1} ; \pi_{n}(X)\right)$ is the cohomology with the local coefficients $\left\{\pi_{n}(X)\right\}$, where $\pi_{1}\left(X_{n-1}\right)=\pi_{1}(X)$ acts on $\pi_{n}(X)$ usually, and $\operatorname{Im} J=\left\{\left[f_{n-1}\right] \in\right.$ $\left.G_{\#}\left(X_{n-1}\right) \mid[k]=f_{n-1}^{*}[k],[k] \in H^{n+1}\left(X_{n-1} ; \pi_{n}(X)\right)\right\}$.

2. Proof of the theorem. Let $X$ be a connected CW-complex and $\left\{X_{n}\right\}$ be a Postnikov-system of $X$.

Let $\phi$ be the usual action of $\pi_{1}\left(X_{1}\right)=\pi_{1}(X)$ on $\pi_{2}(X)$, and put $B=X_{1}=$ $K\left(\pi_{1}(X), 1\right)=K$ in the diagram (1.1). Then we have the following diagram:

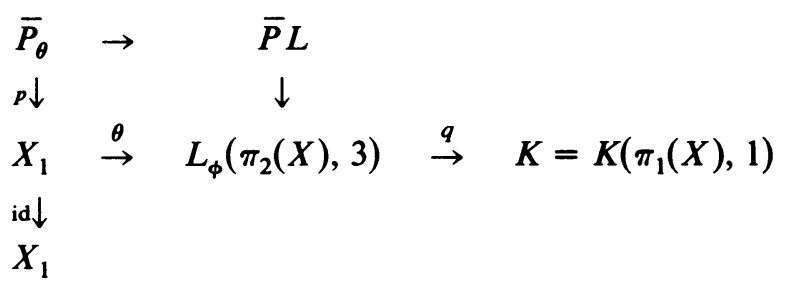

Let $\tau:[F, F]^{\tilde{\phi}} \rightarrow\left[X_{1}, L_{\phi}\left(\pi_{2}(X), 3\right)\right]_{K}=H^{3}\left(X_{1} ; \pi_{2}(X)\right)$ be the transgression. Then we have the following.

Theorem 2.1. If $\theta \in \operatorname{Im} \tau, I\left(1_{\bar{P}_{\theta}}\right)=1$. Therefore, we have the following exact sequence for $\theta \in \operatorname{Im} \tau$.

$$
1=I\left(1_{\bar{P}_{\theta}}\right) \stackrel{\subset}{\rightarrow} H^{2}\left(X_{1} ; \pi_{2}(X)\right) \stackrel{\Delta}{\rightarrow} G_{\#}\left(\bar{P}_{\theta}\right) \stackrel{J}{\rightarrow} G_{\#}\left(X_{1}\right)=1,
$$

where $H^{2}\left(X_{1} ; \pi_{2}(X)\right)$ is the cohomology with the local coefficients $\left\{\pi_{2}(X)\right\}$, where $\pi_{1}\left(X_{1}\right)=\pi_{1}(X)$ acts on $\pi_{2}(X)$ usually.

Proof. The domain of the map $\delta$ is $\left[\bar{P}_{\theta}, \hat{\Omega}_{X_{1}} X_{1}\right]_{X_{1}}$. Since $s=\mathrm{id}, \hat{\Omega}_{X_{1}} X_{1}$ consists of the constant path at every point of $X_{1}$.

Consider the following diagram:

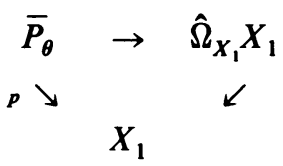

We obtain that $\left[\bar{P}_{\theta}, \hat{\Omega}_{X_{1}} X_{1}\right]_{X_{1}}$ consists of the single element, that is, $\left[\bar{P}_{\theta}, \hat{\Omega}_{X_{1}} X_{1}\right]_{X_{1}}$ $=*$, where $\hat{\Omega}_{X_{1}} X_{1} \rightarrow X_{1}$ is $\omega \rightarrow \omega(p), p=(1,0) \in S^{1}$.

Hence, $\operatorname{Im} \delta=I\left(1_{\bar{P}_{0}}\right)=1$. Since $X_{1}=K\left(\pi_{1}(X), 1\right), G_{\#}\left(X_{1}\right)=1$. Therefore we have the desired sequence. Q.E.D.

Since the identity map $i_{2} \in[F, F]^{\tilde{\phi}}, I\left(1_{X_{2}}\right)=1$. Therefore, we have the following theorem by Proposition 1.5 and the above theorem.

Theorem 2.2. $G_{\#}(X)=H^{j}\left(K\left(\pi_{i}(X), i\right) ; \pi_{j}(X)\right)$ for the connected $C W$-complex $X$ with two nonvanishing homotopy groups $\pi_{i}(X), \pi_{j}(X)(j>i)$, where $\pi_{i}(X)$ acts on $\pi_{j}(X)$ usually if $i=1$.

Furthermore we have the following exact sequence, which is shown by $B$. Schellenberg [9], A. J. Sieradski and M. N. Dyer [3], [4] for the n-dimensional compact connected $\mathrm{CW}$-complex $X$ such that $\pi_{i}(X)=0$ for $1<i<n$. Incidently let $G(X)$ be the group of all based homotopy classes of self-homotopy-equivalences 
of the $n$-dimensional connected CW-complex $X$. Then $G\left(X_{n}\right)=G(X)$. And $X_{n}$ has two nonvanishing homotopy groups, trivially.

Considering the following exact sequence

$$
1 \rightarrow G_{\#}\left(X_{n}\right) \rightarrow G\left(X_{n}\right) \rightarrow \text { Aut } \pi_{1}(X) \times \text { Aut } \pi_{n}(X),
$$

the above theorem implies the following.

TheOREM 2.3. If $X$ is an $n$-dimensional $C W$-complex such that $\pi_{i}(X)=0$ for $1<i<n$, the following sequence is exact:

$$
1 \rightarrow H^{n}\left(\pi_{1}(X) ; \pi_{n}(X)\right) \rightarrow G(X) \rightarrow \text { Aut } \pi_{1}(X) \times \text { Aut } \pi_{n}(X),
$$

where $\pi_{1}(X)$ acts on $\pi_{n}(X)$ in the usual manner.

Moreover, we have the following theorem by Proposition 1.5.

THEOREM 2.4. The following sequence is exact.

$$
1 \rightarrow I\left(1_{X_{3}}\right) \stackrel{\subset}{\rightarrow} H^{3}\left(X_{2} ; \pi_{3}(X)\right) \stackrel{\Delta}{\rightarrow} G_{\#}\left(X_{3}\right) \stackrel{J}{\rightarrow} H^{2}\left(\pi_{1}(X) ; \pi_{2}(X)\right)
$$

and $\operatorname{Im} J=\left\{\left[f_{2}\right] \in G_{\#}\left(X_{2}\right)=H^{2}\left(\pi_{1}(X) ; \pi_{2}(X)\right) \mid[k]=f_{2}^{*}[k], \quad[k] \in\right.$ $\left.H^{4}\left(X_{2} ; \pi_{3}(X)\right)\right\}$.

3. Some examples of $G_{\#}(X)$.

EXAMPLE 3.1 ([2, p. 79]). If $X$ is a connected CW-complex with two nonvanishing homotopy groups $\pi_{1}(X)=G$ (finite cyclic group), $\pi_{n}(X)=M(n>1)$,

$$
G_{\#}(X)= \begin{cases}M^{G} / T M, & n \text { even, } \\ M_{T} / I M, & n \text { odd, }\end{cases}
$$

where $M^{G}$ is the submodule of elements fixed under the action of $G, T M$ is the image of the trace map $T: M \rightarrow M^{G}, M_{T}=\{m \in M \mid T m=0\}$, and, $I M$ is the submodule generated by $\left\{g^{\prime} m \mid g^{\prime} \in I, m \in M\right\}$, where $I$ is the augmentation ideal in the group ring $Z G$.

EXAMPLE 3.2. Every $n$-dimensional cohomology $H^{n}\left(X ; G_{x}\right)$ with the local coefficients $\left\{G_{x}\right\}$ of a connected CW-complex $X$ is isomorphic to $G_{\#}\left(V_{G_{x}}^{n}\right)$ of a suitable $\mathrm{CW}$-complex $V_{G_{x}}^{n}$, where $V_{G_{x}}^{n}$ depends on the dimension $n$ and the local coefficients $\left\{G_{x}\right\}$.

Proof. By [5, Theorem 1.1], we have the following fact, that is, for every path connected space $X$ with base point $*$, there exists a (Serre) fibration $T X \stackrel{t X}{\rightarrow} X$, and the map $t X$ induces an isomorphism on cohomology with local coefficients, i.e., $H^{n}\left(T W ; G_{x}\right)=H^{n}\left(X ; G_{x}\right)$ for every $n>1$, and $\pi_{i}(T X)=0$ for $i>1$.

We can construct a $C W$-complex $V_{G_{x}}^{n}$ such that

$$
\begin{aligned}
& \pi_{1}\left(V_{G_{x}}^{n}\right)=\pi_{1}(T X), \\
& \pi_{n}\left(V_{G_{x}}^{n}\right)=G_{*}, \\
& \pi_{i}\left(V_{G_{x}}^{n}\right)=0 \text { otherwise, }
\end{aligned}
$$

and $\pi_{1}$ action on $G_{*}$ is induced by $\pi_{1}(T X) \stackrel{t X}{\rightarrow} \pi_{1}(X)$.

Trivially, the result is obtained by applying Theorem 2.2. Q.E.D. 


\section{REFERENCES}

1. M. Arkowitz and C. R. Curjel, Groups of homotopy classes, Lecture Notes in Math., vol. 4, Springer-Verlag, Berlin and New York, 1967.

2. A. Babakhanian, Cohomological methods in group theory, Marcel Dekker, New York, 1972.

3. M. N. Dyer, Homotopy classifications of $(\pi, m)$-complexes, J. Pure Appl. Algebra 7 (1976), 249-282.

4. ___ Homotopy trees with trivial classifying ring, Proc. Amer. Math. Soc. 55 (1976), 405-408.

5. D. M. Kan and W. P. Thurston, Every connected space has the homology of a $K(\pi, 1)$, Topology 15 (1976), 253-258.

6. J. F. McClendon, Obstruction theory in fibre spaces, Math. Z. 92 (1971), 1-17.

7. __ Relative principal fibrations, Bol. Soc. Mat. Mexicana 19 (1974), 38-43.

8. Y. Nomura, Homotopy equivalences in a principal fibre space, Math. Z. 92 (1966), 380-388.

9. B. Schellenberg, The group of homotopy self-equivalences of some compact $C W$-complexes, Math. Ann. 200 (1973), 253-266.

10. W. Shih, On the group $\varepsilon[X]$ of homotopy equivalence maps, Bull. Amer. Math. Soc. 70 (1964), 361-365.

11. K. Tsukiyama, Note on self-maps inducing the identity automorphisms of homotopy groups, Hiroshima Math. J. 5 (1975), 215-222.

12. W Note on self-homotopy-equivalences of the twisted principal fibrations, Mem. Fac. Ed. Shimane Univ. Nat. Sci. 11 (1977), 1-8.

Department of Mathematics, Faculty of Education, Shmane University, Matsue, Shmane, JAPAN 